\title{
Dynamics and Control for Surface Exploration of Small Bodies
}

\author{
Julie Bellerose* and Daniel J. Scheeres ${ }^{\dagger}$
}

\begin{abstract}
We provide a general discussion on the surface dynamics of particles in the gravitational field of a small body system. In this paper, we model the asteroid as an ellipsoid, and also consider binary systems. We investigate the dynamics near the stable and unstable surface equilibria of an ellipsoid body under different perturbations. We give analytical tools to approximate the total distance covered and the amount of time it takes for a particle to settle on a non-ideal surface. Using these analytical tools, we develop control laws for single and multiple collaborating vehicles to counteract the effect of surface equilibria. Simulations of the dynamics and control are shown.
\end{abstract}

\section{Introduction}

Previous work have characterized the dynamics of asteroids and binary asteroid systems orbiters. $^{2,6,13}$ A number of missions have flown by or have been sent to small bodies in order to investigate their composition. Only a few of these missions have landed on their surface. Although the NEAR mission still sits on the surface of Eros, the Japanese spacecraft Hayabusa is currently the only one that carried a surface lander. ${ }^{14}$ The autonomous rover had a torque system to make it move on the surface, ${ }^{8}$ where the decision to move was made using photo diodes to know whether the rover was standing on the surface or not. A scheme was also implemented for the rover to move away or toward the sunlight depending whether it was morning or evening on the asteroid. ${ }^{14}$

To date, analytical methods on the dynamics and control of surface explorers are limited. Earlier work has looked at asteroid and comet ejecta. ${ }^{12}$ The surface equilibria of small bodies have been studied for rotating ellipsoids, ${ }^{5}$ where the authors looked at surface equilibria and their stability as function of the ellipsoid parameters and spin. Although some observations have been made on accumulations of dust and particles following these stability properties on small bodies, no studies have looked at the general surface motion for exploration purposes. This paper links surface equilibrium properties and general surface motion. From simulations, we can see that these stability regions have some effects on the dynamics of moving objects, where a particle stays close to a stable surface equilibrium, and move away from an unstable one.

However, not having access to some regions of an asteroid may lead to mission failures. Thus, in order to develop control methods, we derive a prediction dynamical model to estimate the travel distance and the time taken to reach a stop. A control law derived from sliding mode control is then applied. And to allow a variety of mapping and sampling applications, the control method is further extended to a collaborative formation of three landers.

${ }^{*}$ Research Assistant, University of Michigan, Ann Arbor, MI 48109, juliebel@umich.edu.

${ }^{\dagger}$ A. Richard Seebass Endowed Chair Professor, Colorado Center for Astrodynamics Research, Department of Aerospace Engineering Sciences, The University of Colorado at Boulder, 80309, scheeres@colorado.edu, Member AAS, Associate Fellow AIAA. 


\section{Dynamics of Asteroids}

In order to simulate surface motion, it is necessary to account for the dynamics of asteroids. As shown in Figure 1, we model the asteroid as a tri-axial ellipsoid. The potential of an ellipsoidal

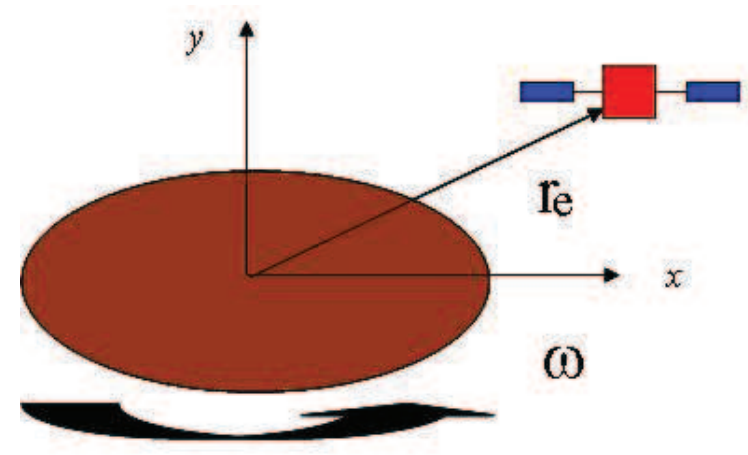

Figure 1. Representation of the asteroid orbiter problem.

body is written in terms of elliptic integral. ${ }^{3,4}$ We can write the dynamics of a spacecraft in an asteroid fixed frame as

$$
\ddot{\boldsymbol{r}}_{\boldsymbol{e}}+2 \boldsymbol{\omega} \times \dot{\boldsymbol{r}}_{\boldsymbol{e}}+\boldsymbol{\omega} \times\left(\boldsymbol{\omega} \times \boldsymbol{r}_{\boldsymbol{e}}\right)=\frac{\partial U_{e}}{\partial \boldsymbol{r}_{\boldsymbol{e}}}=\frac{3}{4} \int_{\lambda}^{\infty} \phi(\boldsymbol{r}, v) \frac{d v}{\triangle(v)},
$$

where $U_{e}$ has the form

$$
U_{e}=\frac{3}{4} \int_{\lambda}^{\infty} \phi(\boldsymbol{r}, v) \frac{d v}{\Delta(v)}
$$

with

$$
\phi(\boldsymbol{r}, v)=1-\frac{(x+\nu r)^{2}}{1+v}-\frac{y^{2}}{\beta^{2}+v}-\frac{z^{2}}{\gamma^{2}+v}
$$

and

$$
\Delta(v)=\sqrt{(1+v)\left(\beta^{2}+v\right)\left(\gamma^{2}+v\right)},
$$

where $0<\gamma \leq \beta \leq 1, \gamma$ and $\beta$ correspond to the $z$ and $y$ radii of the ellipsoid, and $\lambda$ satisfies $\phi(\boldsymbol{r}, \lambda)=0$. This system also allows for one integral of motion, the Jacobi integral, written as

$$
J=\frac{1}{2}\left(\dot{x}^{2}+\dot{y}^{2}+\dot{z}^{2}\right)-\frac{1}{2} \omega^{2}\left(x^{2}+y^{2}\right)-U_{e} .
$$

The dynamics for asteroid systems with more than one bodies are expressed in a different form. Studies have looked at the dynamics of two-body systems ${ }^{1,3,7,9,10}$ and the dynamics of a spacecraft close to them. ${ }^{2,11}$ A useful simplification is to let one of the bodies be a sphere. In this case, the equations of motion of the spacecraft are written as

$$
\ddot{\tilde{\boldsymbol{\rho}}}+2 \boldsymbol{\omega} \times \dot{\tilde{\boldsymbol{\rho}}}+\boldsymbol{\omega} \times(\boldsymbol{\omega} \times \tilde{\boldsymbol{\rho}})=\frac{\partial U_{12}}{\partial \tilde{\boldsymbol{\rho}}},
$$

where $U_{12}$ is the potential from both bodies, $\omega$ is the angular velocity of the non-spherical body. We use $\boldsymbol{\rho}$ for the nondimensional position vector of the spacecraft relative to the binary system center of mass, in a frame fixed to the ellipsoid. 


\section{Surface Motion}

\section{A. Hopping on a Flat Surface}

As shown in Figure 2, a particle impacting on a flat surface has an incoming velocity $v_{0}$ at an angle $\alpha_{0}$. As a first step, the impact can be modeled as a perfectly inelastic impact with no sliding, that is $c_{r}=0$ and $\mu=\inf$, where $c_{r}$ and $\mu$ are the coefficient of restitution and friction. This results in the particle staying at the same location when touching the surface, or getting "stuck" at impact. On the other hand, one can look at the ideal elastic collision with $c_{r}=1$. In this case, a particle impact has no loss associated with it and the normal velocity is reversed in direction but keeps the same magnitude. If there is no impulse or friction, the tangential direction is unchanged.

However, it is more likely that the particle will slightly deform from the impact with the asteroid, having a coefficient of restitution between 0 and 1. And to better model an asteroid surface, the contact between the two objects will have a finite coefficient of friction, $\mu$, adding an impulse opposite to the direction of motion. With a coefficient of restitution $c_{r}$ and friction force $\mu N$ at the impact point, where $N$ is the normal reaction force, the normal and tangential components of the velocity after impact are,

$$
\begin{gathered}
v_{n}^{1}=c_{r} v_{n 0}=c_{r} v_{0} \sin \left(\alpha_{0}\right) \\
v_{T}^{1}=v_{0} \cos \left(\alpha_{0}\right)-\mu\left(1+c_{r}\right) v_{0} \sin \left(\alpha_{0}\right)
\end{gathered}
$$

Therefore, the velocity after impact has a new direction, $\alpha_{1}$,

$$
\tan \alpha^{1}=\frac{v^{1} n}{v^{1} T}=\frac{c_{r} \sin \left(\alpha_{0}\right)}{\cos \left(\alpha_{0}\right)-\mu\left(1+c_{r}\right) \sin \left(\alpha_{0}\right)}
$$

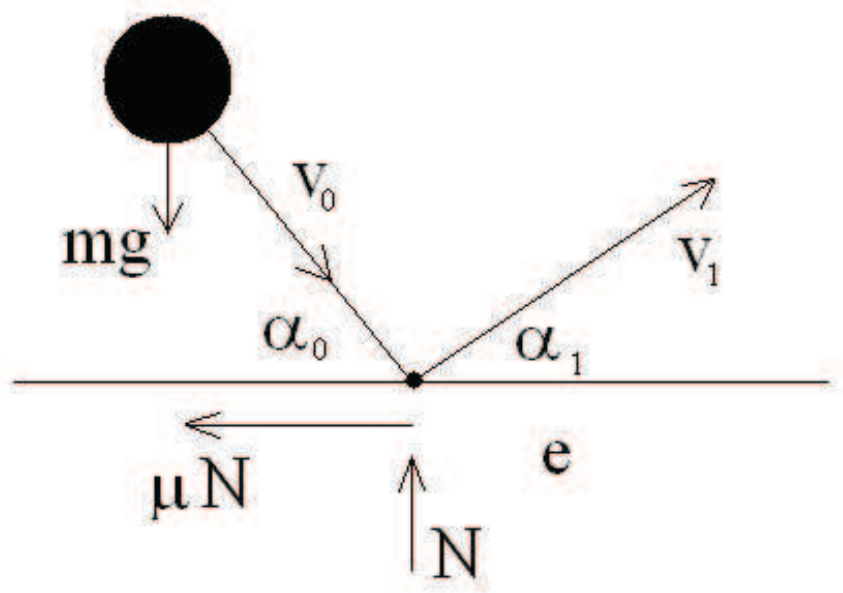

Figure 2. Dynamics of collisions for a particle on a flat surface with restitution and friction coefficient $c_{r}$ and $\mu$ respectively.

Using impacts dynamics, it is possible to estimate the total time and distance from an initial jump to a stop due to friction and restitution coefficients. A special attention is needed to model the surface of an asteroid, and to take into account the non-uniform gravity field of a binary system.

\section{B. Surface Modeling}

To estimate the distance reached and time of travel on a curved surface, we define a coordinate frame fixed at the initial impact point on the surface, as shown on Figure 3. In this frame, the 
normal is defined as being the unit vector in the direction of the surface gradient,

$$
\hat{\boldsymbol{n}}=\frac{\nabla S}{|\nabla S|}
$$

where $S$ represents the ellipsoid surface expressed as $S=x^{2}+\frac{y^{2}}{\beta^{2}}+\frac{z^{2}}{\gamma^{2}}-1=0$. The tangential direction is then defined as the unit vector perpendicular to the velocity,

$$
\hat{t}=\frac{\hat{t} \times V}{|\hat{t} \times V|},
$$

where $\boldsymbol{V}$ is the impact velocity expressed in the body fixed frame. The cross track unit vector is then obtained from orthogonality of the two first unit vectors,

$$
\hat{\boldsymbol{d}}=\hat{\boldsymbol{t}} \times \hat{\boldsymbol{n}} .
$$

Note that the cross track unit vector is always tangent at the impact point in the direction of the velocity, but that the total acceleration can act in an arbitrary direction. For numerical simulations,

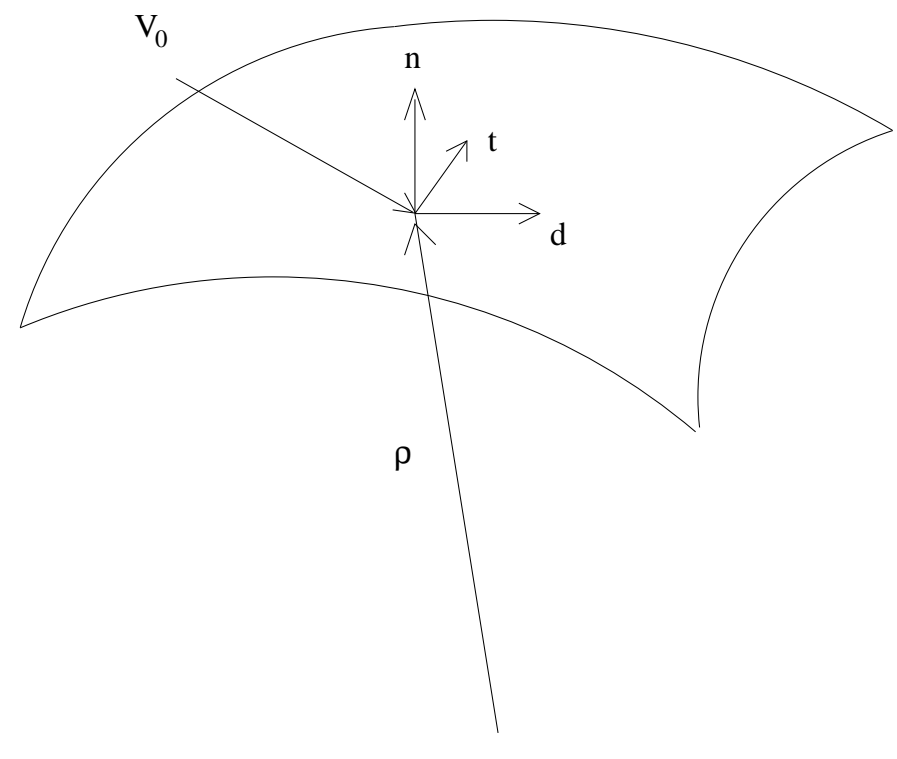

Center of ellipsoid

Figure 3. Geometry for 3D dynamical model of surface landers on small bodies.

the velocity components computed after impact can be converted back to the asteroid system fixed frame and integrated until the next impact.

\section{Effect of a Non-Uniform Gravity Field}

While the surface modeling above can be applied on the surface of any single asteroids or binary asteroid systems, the major difference is in the gravitational field. The general assumption for impacts on a flat surface, as shown in Figure 2 is that the particle moves in a uniform gravity field. For a non flat surface, the gravity vector is dependent on the mass distribution of the body. For a sphere, the gravitational field is equivalent to the one of a point mass. ${ }^{3}$ In the case of an ellipsoid, the gravitational field is not uniform, as shown in Figure 4. Note that the gray corresponds to the 


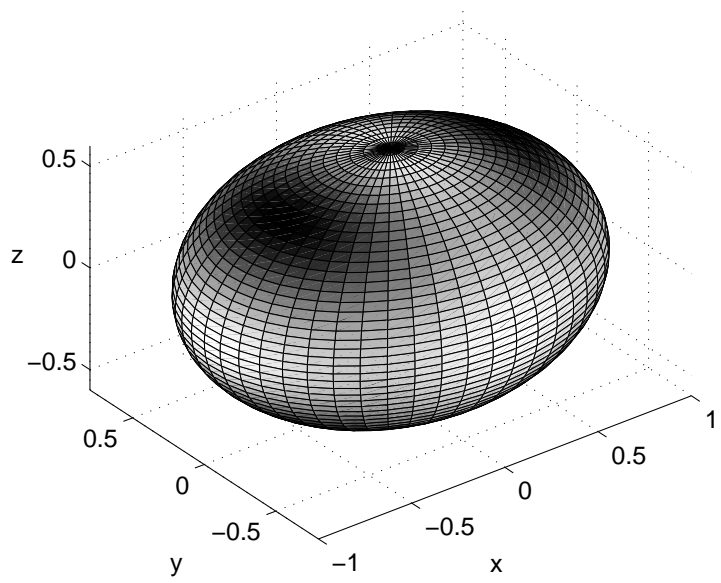

Figure 4. Gravity field of an ellipsoidal body. The gray scale corresponds to the deviation of the gravity vector from the centroid, where the dark regions have the largest deviations.

deviation of the gravity vector from the centroid, where the dark regions have the largest deviations. The ellipsoid has parameters $[\alpha ; \beta ; \gamma]=[1 ; 0.8 ; 0.6]$.

The case of a binary system is shown in Figure 5. Note that only the ellipsoid is shown. In this case, the binary has a mass fraction of 0.95 , with a distance of 9 units between the bodies and ellipsoid parameters of $[\alpha ; \beta ; \gamma]=[1 ; 0.8,0.6]$. The gray corresponds to the deviation of the gravity vector from the ellipsoid centroid, where the dark regions have the largest deviations. The direction

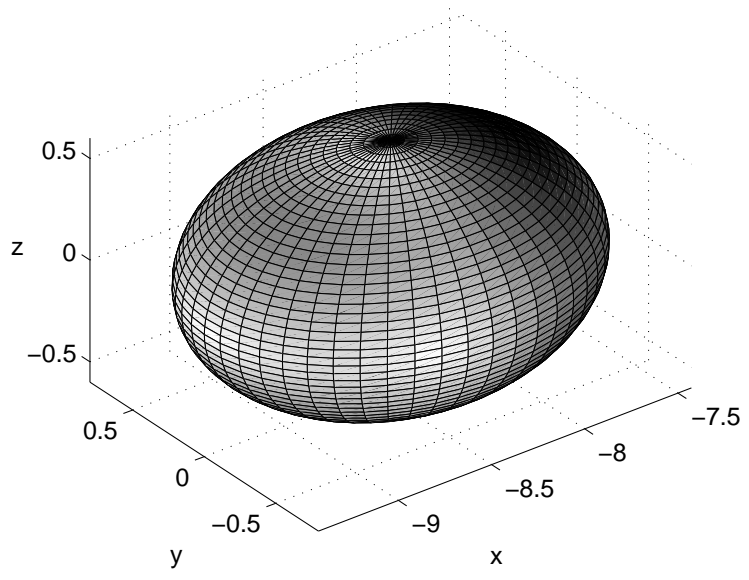

Figure 5. Gravity field of a binary system. The gray scale corresponds to the deviation of the gravity vector from the centroid, where the dark regions have the largest deviations.

of the combined gravity field is found using the equations of motion given by Eq. (6). The following analytical method is developed for approximating the dynamics on a curved surface, under a nonuniform gravity field. The method approximates the local surface as a flat surface, taking the initial gravity vector as constant over that surface. For numerical simulations, the gravity vector needs to be calculated at every point of impact. Note that the gravity vector needs to be transformed to 
the surface frame defined in the previous section.

\section{Analytical Model for Dynamics on a Curved Surface in a Non-Uniform Gravity Field}

Having defined a surface frame, the geometry for modeling the surface dynamics is described in Figure 6. At the impact point, the lander is subjected to a local coefficient of restitution and surface friction factor, $c_{r}$ and $\mu$ respectively. The incoming velocity $\boldsymbol{v}$, just before the next impact, is influenced by the gravity vector $\boldsymbol{g}$ and the rotational acceleration. Note that the geometry is shown for a bounce in the normal - cross track plane, $\hat{\boldsymbol{n}}-\hat{\boldsymbol{d}}$. By approximating the local surface as a flat surface, that is, considering the motion on a local tangent plane at the point of impact, it is possible to find general expressions for the time, distance and velocity components between jumps as function of the initial velocity and gravity vectors.

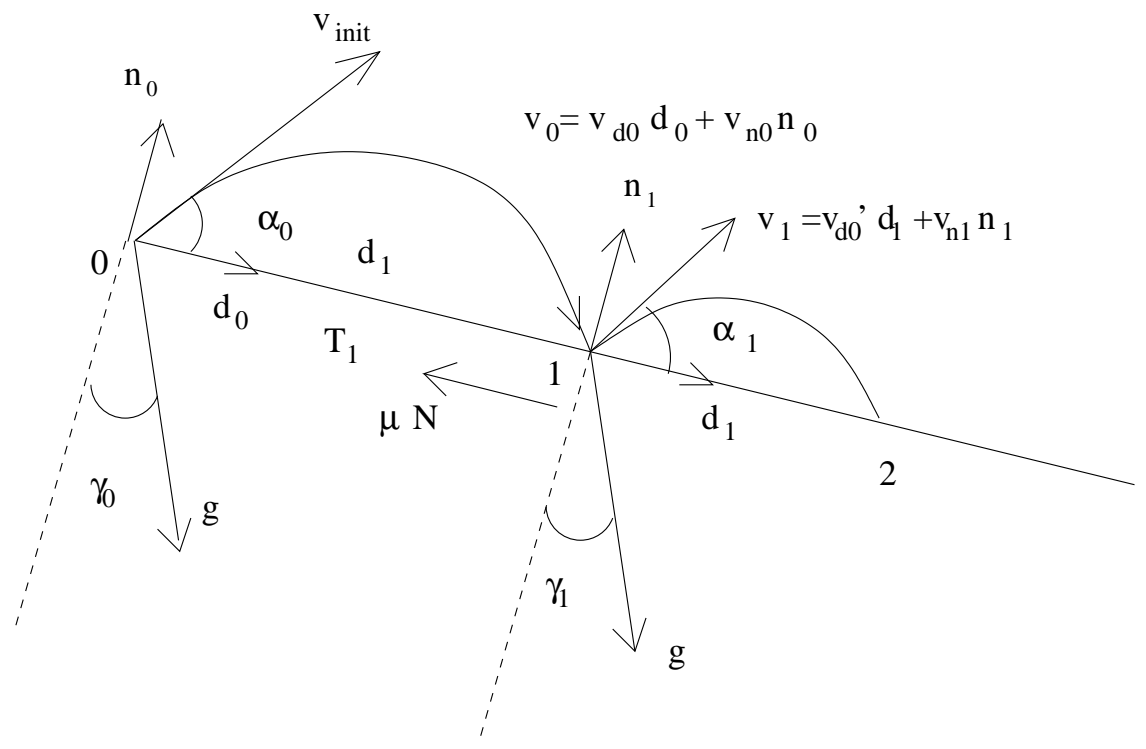

Figure 6. Dynamics of collisions for a particle on an inclined surface with restitution and friction coefficient $e$ and $\mu$ respectively.

The normal component of the velocity after impact is

$$
v_{n_{1}}=-c_{r} v_{n_{0}}
$$

where $v_{n_{0}}$ and $v_{n_{1}}$ are the normal components before and after impact, respectively. In the tangential direction, the velocity expression is

$$
v_{d_{0}}^{\prime}=v_{d_{0}}-\mu\left(v_{n_{0}}+v_{n_{1}}\right)=v_{d_{0}}-\mu\left(1+c_{r}\right) v_{n_{0}},
$$

where $v_{d_{0}}^{\prime}$ and $v_{d_{0}}$ are the tangential components before and immediately after impact, respectively. When the particle arrives at the next impact, it is influenced by gravity. Hence, the change in the cross track velocity $v_{d_{0}}$ is written as

$$
v_{d_{0}}=v_{0_{d}}+g_{d_{0}} t_{01}
$$

with

$$
t_{01}=\frac{2 v_{n_{0}}}{g_{n_{0}}}
$$


and where $g_{n_{0}}$ and $g_{d_{0}}$ are the normal and cross track components of the initial gravity vector.

It is possible to find general expressions for the velocities before and after impacts. In vector form, the velocity after the $n+1^{\text {th }}$ impact is expressed as

$$
\boldsymbol{V}_{\boldsymbol{n}+\mathbf{1}}^{\prime}=-c_{r} \hat{\boldsymbol{n}} \hat{\boldsymbol{n}} \cdot \boldsymbol{V}_{\boldsymbol{n}}+\left(1-\mu\left(1+c_{r}\right)\left(\hat{\boldsymbol{n}} \cdot \boldsymbol{V}_{\boldsymbol{n}}\right)\right)[\boldsymbol{U}-\hat{\boldsymbol{n}} \hat{\boldsymbol{n}}] \cdot V_{\boldsymbol{n}},
$$

where $\boldsymbol{U}$ is the unity dyad. Similarly, the velocity just before the $n+1^{\text {th }}$ impact is given by

$$
\boldsymbol{V}_{\boldsymbol{n}+\mathbf{1}}=-c_{r} \hat{\boldsymbol{n}} \hat{\boldsymbol{n}} \cdot \boldsymbol{V}_{\boldsymbol{n}}+\left(1-\mu\left(1+c_{r}\right)\left(\hat{\boldsymbol{n}} \cdot \boldsymbol{V}_{\boldsymbol{n}}\right)\right)[\boldsymbol{U}-\hat{\boldsymbol{n}} \hat{\boldsymbol{n}}] \cdot \boldsymbol{V}_{\boldsymbol{n}}+\Delta t[\boldsymbol{U}-\hat{\boldsymbol{d}} \hat{\boldsymbol{d}}] \cdot \boldsymbol{g},
$$

where

$$
\Delta t=\frac{2\left|v_{n_{0}}\right|}{g_{n_{0}}} .
$$

Then, the distance covered between the $n^{\text {th }}$ and $n+1^{\text {th }}$ hop is expressed as,

$$
d_{n, n+1}=v_{t_{n-1}}^{\prime} t_{n, n+1}+\frac{1}{2} g \sin \gamma_{0} t_{n, n+1}^{2} .
$$

The time of travel and the distance covered can be estimated using summations of the interval expressions given by Eqs. (19-20). The time for the rover to reach a stop is given by

$$
t_{\infty}=\frac{2 v_{n_{0}}}{g_{n_{0}}}\left(\frac{1}{1-c_{r}}\right)
$$

Similarly, the distance traveled is

$$
d_{d, \infty}=\frac{2 v_{n_{0}} v_{0_{d}}}{g_{n_{0}}\left(1-c_{r}\right)}+\frac{2 v_{n_{0}}^{2}}{g_{n_{0}}\left(1-c_{r}\right)^{2}}\left(-\mu c_{r}+\frac{g_{d_{0}}}{g_{n_{0}}}\right)
$$

Equations (21-22) should agree with numerical simulations for small hops. Errors may come from tangential deviation due to the non-uniform gravity vector and the effect of the rotating body which is not accounted for in the analytical result.

\section{E. Validation and Numerical Analysis of Surface Motion}

In is interesting to look into a range of hops for validation and to investigate the effect of surface parameters and hopping conditions. Figure 7 shows an ideal case of surface hopping, where the surface is frictionless and under elastic impacts. Typical values for these surface parameters are close to semisolid environment, with surface made of dirt and some loose gravel. ${ }^{8,14}$ The resulting motion is damped compared to the ideal case shown in Figure 7. In Figure 8, the particle moves along the equator on a surface having a friction coefficient of 0.1 and 0.5 . Adding a $75 \%$ non-ideal coefficient of restitution can make an impact loose more than half of its initial height. In addition, Figure 9 shows the effect of different initial velocities, and launching from different angles. For these simulations, the surface was modeled with a restitution of 0.25 and a friction factor of $\mu=1$. As expected, the motion damps quickly.

For the numerical cases shown in Figures 7-9, the total time is calculated using the numerical integrator scheme, adding every interval of time between hops. Similarly, the total distance traveled can be calculated, accounting for the curvature of the ellipsoid in this case. In order to validate the method developed using the analytical surface dynamics, these values of time and distance are compared to Eqs. (21) and (22). It was found that, provided the jumps don't exceed 5 meters, the analytical model agree within $1 \%$ compared to the numerical simulations. These simulations were done for an asteroid size of $600 \mathrm{~m} \times 500 \mathrm{~m} \times 300 \mathrm{~m}$. 


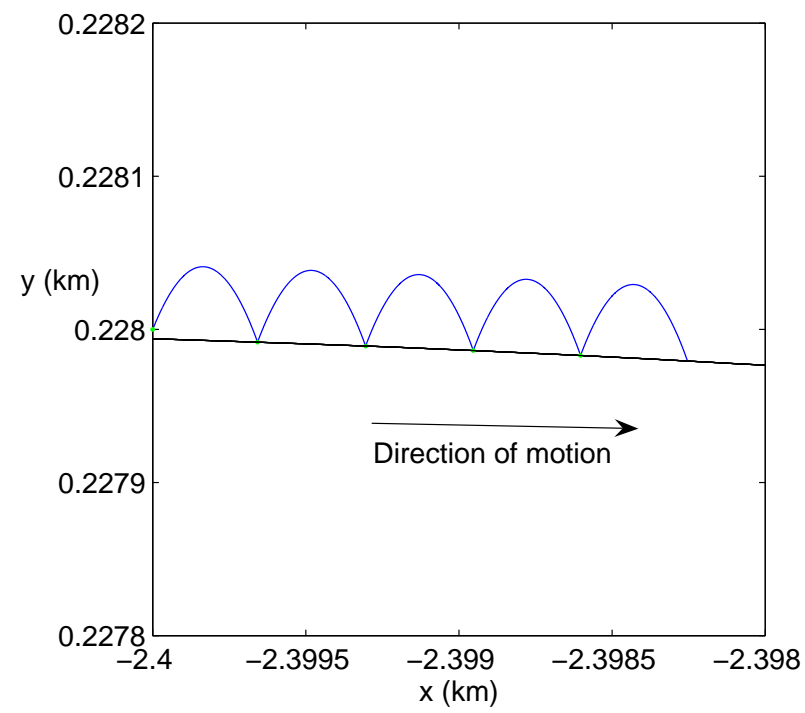

Figure 7. Elastic impacts for a particle moving on the surface of an ellipsoid.

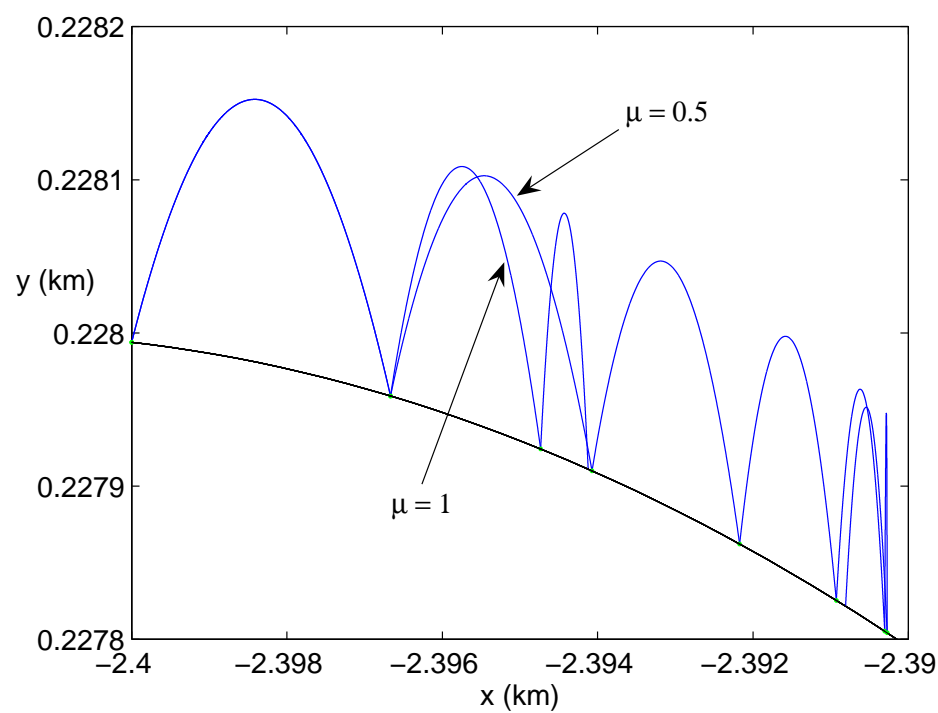

Figure 8. Top view of a particle moving along equator for perfectly elastic impacts under friction of 1 and 0.5 . 


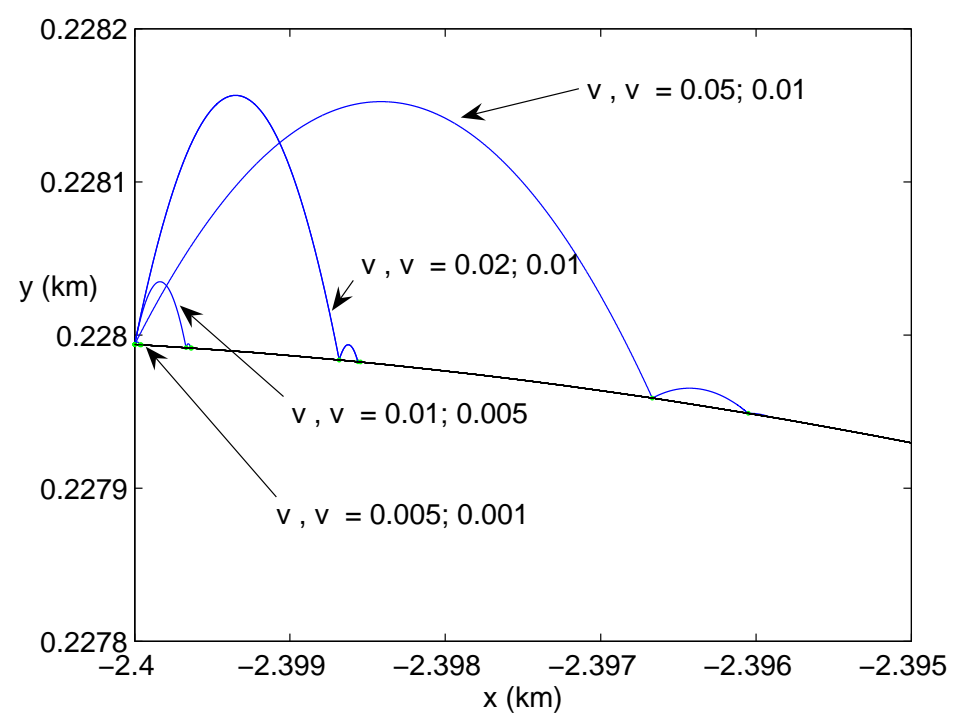

Figure 9. Effect of initial velocities on surface motion considering a surface modeled with a restitution and friction coefficients of 0.25 and 1 , respectively.

\section{F. Condition for Zero Velocity}

Mathematically, friction could overcome the transversal velocity at impact and make the resulting transversal velocity to be negative, which is physically impossible. In reality, a negative transverse velocity will lead to a lateral stop. Figure 8 shows simulations for varying friction factors. We see clearly that adding more friction slows down any dynamical motion rapidly.

For arbitrary initial conditions, the number of bounces can be computed in order for a vehicle to reach a stop. If $N$ is the stopping bounce, then $v_{t_{N-1}}^{\prime}=0$. We want to compute the condition from Eq. (17) such that,

$$
v_{0_{d}}-\frac{\mu v_{N_{0}}\left(1+c_{r}\right)\left(1-c_{r}^{N}\right)}{\left(1-c_{r}\right)}+\frac{2 v_{N_{0}} g_{d_{0}}\left(1-c_{r}^{N}\right)}{g_{n_{0}}\left(1-c_{r}\right)} \leq 0
$$

Note that the particle may still have a normal velocity. We compute $N$ from the condition that

$$
v_{0_{d}} \leq\left[\mu\left(1+c_{r}\right)-2 \frac{g_{d_{0}}}{g_{n_{0}}}\right] \frac{v_{N_{0}}\left(1-c_{r}^{N}\right)}{\left(1-c_{r}\right)},
$$

with the assumption that $\left[\mu\left(1+c_{r}\right)-2 \frac{g_{d_{0}}}{g_{n_{0}}}\right]>0$ for $N=1$. It can be shown that

$$
N \geq \frac{\ln \left[1-\frac{v_{0_{d}}}{v_{N_{0}}} \frac{\left(1-c_{r}\right)}{\left(\mu\left(1+c_{r}\right)-2 \frac{g_{d_{0}}}{g_{n}}\right)}\right]}{\ln c_{r}} .
$$

Having defined all mathematical tools for this analytical method to be valid and consistent, the next step is to look at the dynamics on the surface of a rotating body.

\section{G. Influence of a Rotating Ellipsoid on the Surface Dynamics}

On a rotating ellipsoid, a particle is influenced by the Coriolis and centripetal accelerations. As shown in Figure 10, on the side leading the rotational motion denoted by $A$, both the centripetal 
and Coriolis accelerations are pointed away from the body. The particle on the surface would then be assisted in its jump, allowing to cover a longer distance. On the other side, at $B$, the two accelerations are opposite to each other, which causes the particle to slow down.

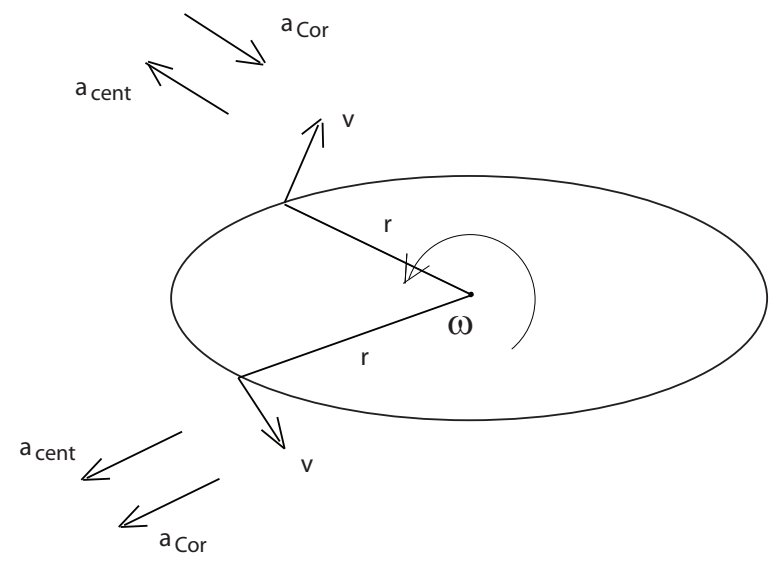

Figure 10. Influence of the coriolis and centripetal accelerations.

It is possible to find points on an ellipsoid surface where a particle could stay in equilibrium. The stability of these equilibrium points depends on the ellipsoid spin and shape parameters. As a result, it is expected that the dynamics of particles are influenced by these equilibria and their stability.

The dynamics on the surface of a single rotating ellipsoid can be written using Lagrange coefficients, ${ }^{5}$

$$
\ddot{\overline{\boldsymbol{\rho}}}+2 \boldsymbol{\omega}_{\boldsymbol{r}} \times \dot{\overline{\boldsymbol{\rho}}}+\omega_{\boldsymbol{r}} \times\left(\boldsymbol{\omega}_{\boldsymbol{r}} \times \overline{\boldsymbol{\rho}}\right)=\frac{\partial U_{e}}{\partial \overline{\boldsymbol{\rho}}}+\lambda \nabla S,
$$

where $\overline{\boldsymbol{\rho}}$ is the nondimensional position vector of a particle on the surface relative to the ellipsoid center of mass, $\boldsymbol{\omega}_{\boldsymbol{r}}$ is the ellipsoid spin, and $U_{e}$ is the ellipsoid potential, as defined by Eqs. (2-4). Three equilibrium points are found, one at each axis on the ellipsoid. Their stability can also be investigated using classical dynamics and geometrical analysis. ${ }^{5}$

Simulations show that, for small perturbations, a moving object on the surface of a rotating ellipsoid tends to stay closer to a stable equilibrium point, and tend to stay further away from an unstable one. Figure 11 shows this dynamics. In this case, a particle is being dropped near an unstable pole, and moves toward the stable point along the $y$ axis of the ellipsoid $\left(P_{2}\right)$. Note that the simulations are obtained for an ideal surface. Figures 12 and 13 show examples of the dynamics when the polar regions are stable. The curves shown are made of a series of hops under perfect surface conditions, with smaller disturbances in Figure 13. Figure 14 shows a simulation of surface dynamics for the same ellipsoid but under non ideal conditions, with restitution and friction coefficients of 0.5 . In this case, it is clear that the general surface dynamics are influenced by the stability of the polar region.

An ellipsoid rotating at a fast spin rate or a more prolate ellipsoidal body are typical cases to have stable surface equilibria at the equator instead of at the polar regions. A particle placed at the same relative latitude as in Figure 14 will tend to stay away from the unstable polar region, and, instead, go toward the equator, which is represented in Figure 15. Extending this study to robotic surface explorers, the influence of surface equilibria on their dynamics provides a motivation to develop control methods to allow surface exploration of specific regions on asteroids. 


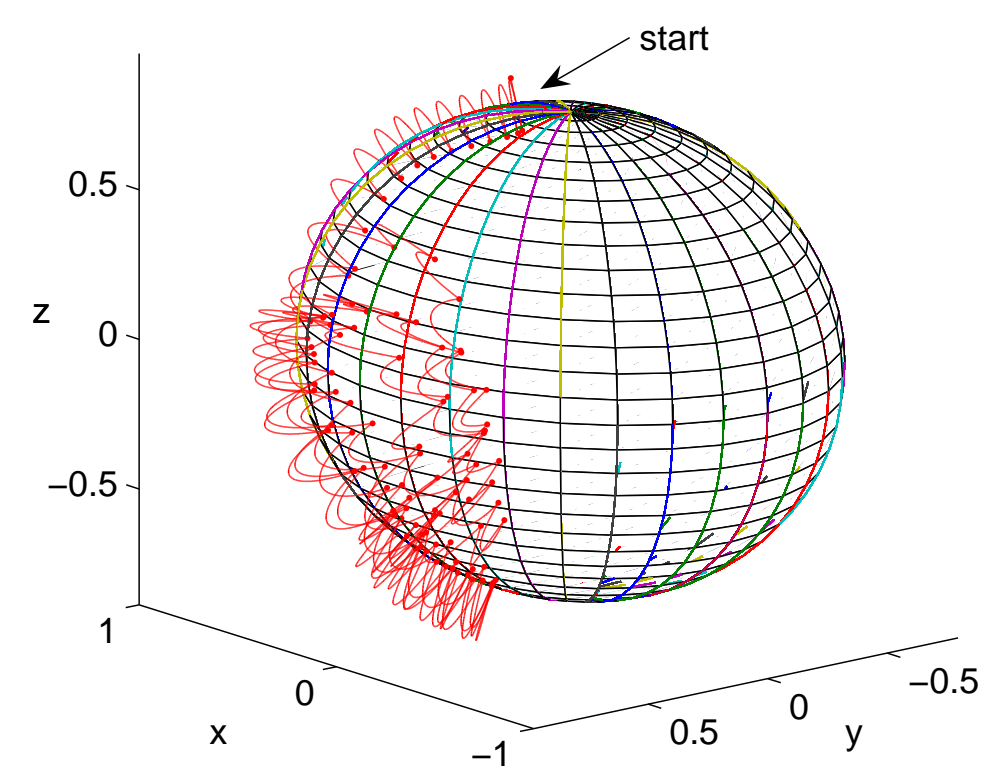

Figure 11. Dynamics around stable and unstable points of a rotating ellipsoid. The point along the $y$-axis, $P_{2}$, is stable.

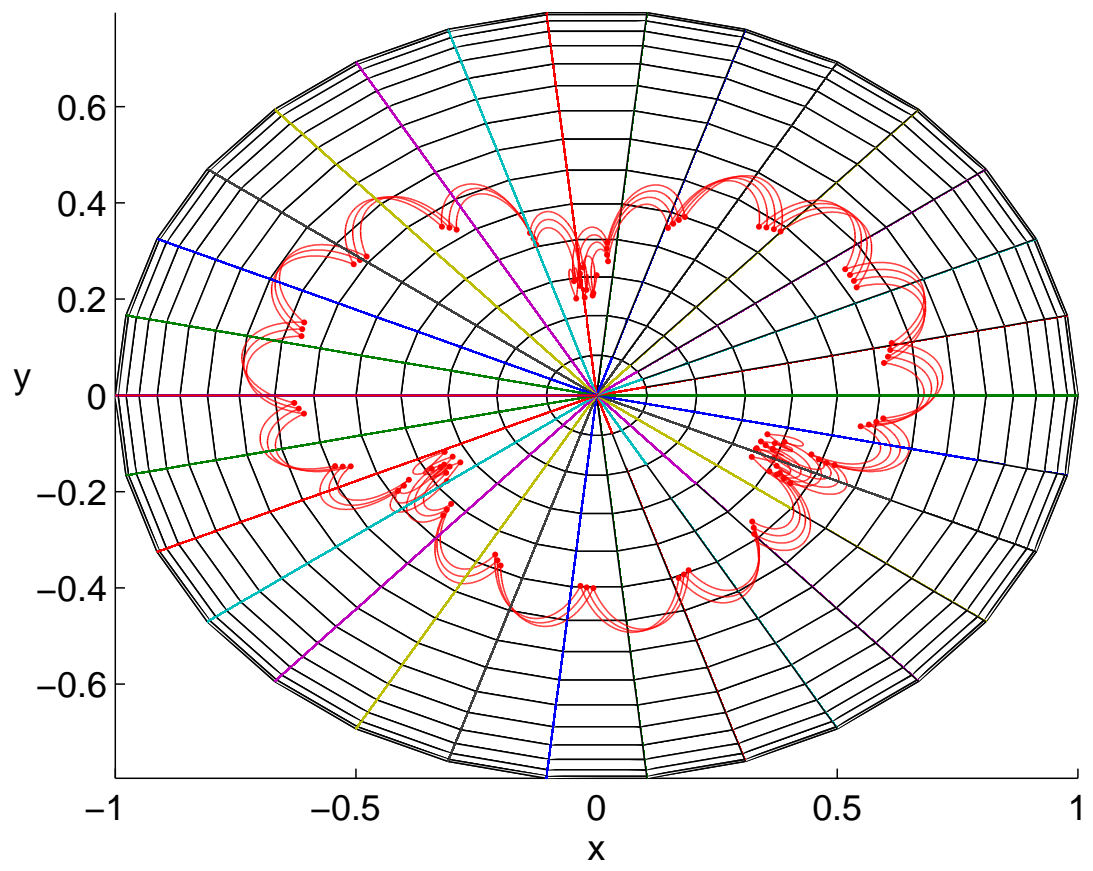

Figure 12. Dynamics around the stable polar point of a rotating ellipsoid, $P_{3}$. 


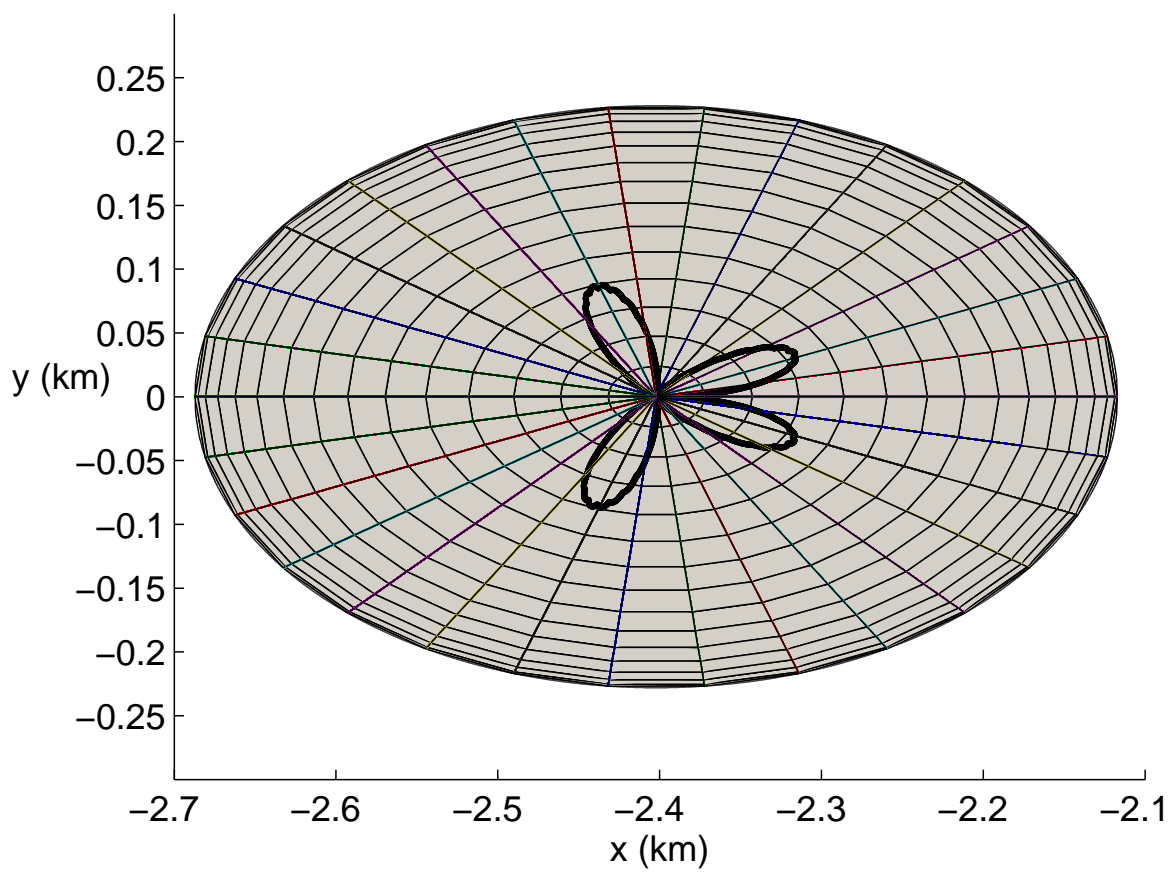

Figure 13. Top view of the dynamics close to a stable pole. The trace is made of hops under ideal conditions with zero friction and elastic impacts.

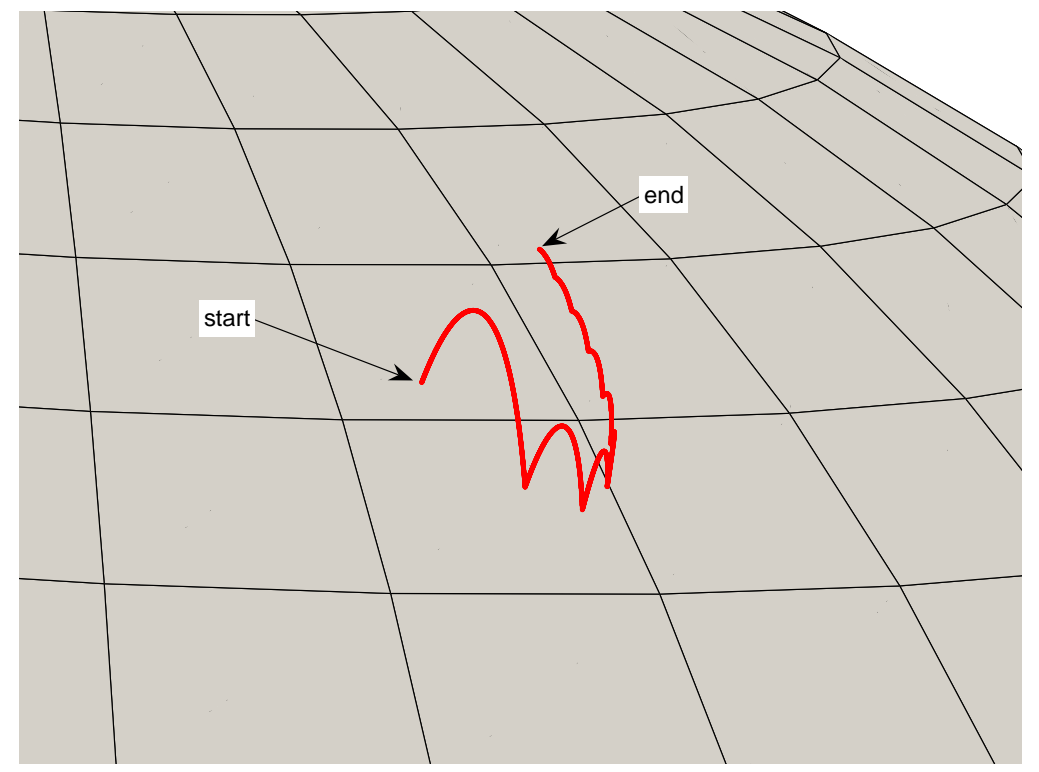

Figure 14. Dynamics close to a stable pole assuming restitution and friction factors of 0.5. 


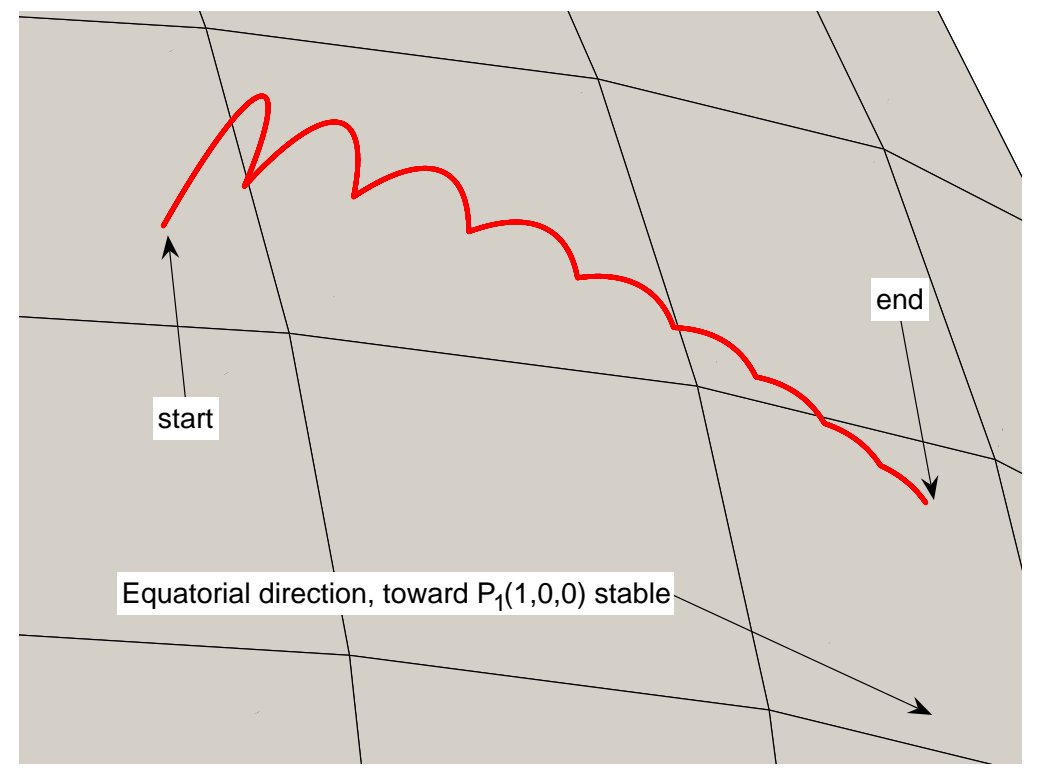

Figure 15. Dynamics close to a stable equatorial axis assuming restitution and friction factors of 0.5 .

\section{Control Algorithms}

\section{A. Control Law for a single lander (hopper)}

A discrete control law can be developed to control surface motion based on the analytical dynamics discussed. The idea is derived from sliding mode control used for Unmanned Aerial Vehicle (UVA). ${ }^{15}$ If the position at the $n^{\text {th }}$ jump is represented in the cross track-tangential plane, $\hat{\boldsymbol{d}}-\hat{\boldsymbol{t}}$, by

$$
\eta^{n}=\left[\begin{array}{l}
d_{d} \\
d_{t}
\end{array}\right]
$$

The $n+1^{\text {th }}$ location is then the sum of the previous location and the distance covered from jumping

$$
\eta^{n+1}=\eta^{n}+\Delta \eta
$$

where $\Delta \eta$ is to be solved in such a way that the error on the position decreases in time. If the error at the $n^{\text {th }}$ jump is defined as

$$
\epsilon^{n}=\eta^{n}-\eta_{d},
$$

where $\eta_{d}$ is the desired end position, then a control parameter $K$ can be chosen such that the error decreases over each bounce, that is,

$$
\epsilon^{n+1}=e^{-K} \epsilon^{n}
$$

Substituting for $\epsilon^{n+1}$ and $\epsilon^{n}$ in Eq. (30) gives

$$
\eta^{n+1}-\eta_{d}=\left(\eta^{n}-\eta_{d}\right) e^{-K}
$$

Or, substituting Eq. (28) and solving for $\Delta \eta$ give

$$
\Delta \eta=\left(\eta^{n}-\eta_{d}\right)\left[e^{-K}-1\right] .
$$


$\Delta \eta$ is then the distance to be covered given a desired position and control parameter $K$. Using this result in the surface dynamics model, Eq. (22) can be inverted to find the initial velocity component. Hence, the cross track velocity component is given by

$$
v_{0_{d}}=\frac{g_{n_{0}}\left(1-c_{r}\right)}{2 v_{n_{0}}}\left[d_{d}+\frac{2 v_{n_{0}}^{2} \mu c_{r}}{g_{n_{0}}\left(1-c_{r}\right)^{2}}+\frac{2 v_{n_{0}}^{2} g_{t_{0}}}{g_{n_{0}}^{2}\left(1-c_{r}\right)^{2}}\right] .
$$

Equation (33) is used to compute the initial velocities in order to achieve the distance $\Delta \eta$. Under the same surface conditions, we note that increasing the control parameter reduces the number of hops necessary to reach a desired position, to the point where the lander can easily overshoot its target. A more efficiency strategy may be to undershoot the target as, for small hops, a hopper would only need to re-estimate the necessary initial conditions to reach the end point within an acceptable bound.

\section{B. Extension to Cooperative Rovers}

To opening up to a variety of applications, the control method was extended to collaborative hoppers, with the goal of navigating a formation to a desired end point. For collaborative landers, the notation $\eta_{i}^{n}$ is used for the $i^{t h}$ hopper at the $n^{\text {th }}$ location. The $n+1^{t h}$ location is then defined as

$$
\eta_{i}^{n+1}=\eta_{i}^{n}+\Delta \eta_{i}
$$

where, again, the distance, $\Delta \eta_{i}$, is solved in order to decrease the error in time. For this application, the method needs to take into account the error on a hopper's desired location, and their relative error. The error term is then defined as,

$$
\begin{aligned}
\epsilon_{i}^{n}= & \left(\eta_{i}^{n}-\eta_{d, i}^{n}\right)+K_{r}\left(\eta_{i, j}^{n}-\eta_{i-1, j}^{n}+\eta_{d, i-1, j}\right) \\
& +K_{r}\left(\eta_{i, j}^{n}-\eta_{i+1, j}^{n}+\eta_{d, i+1, j}\right)+K_{r}\left(\eta_{i, j}^{n}-\eta_{i, j-1}^{n}+\eta_{d, i, j-1}\right),
\end{aligned}
$$

where $K_{r}$ is a weight factor giving precedence on the absolute or relative hopper end position.

Earlier work has shown mesh stability of a triangular formation as shown on Figure $16 .^{15}$ Simulations were done for three hoppers. As for the single hopper, we still want

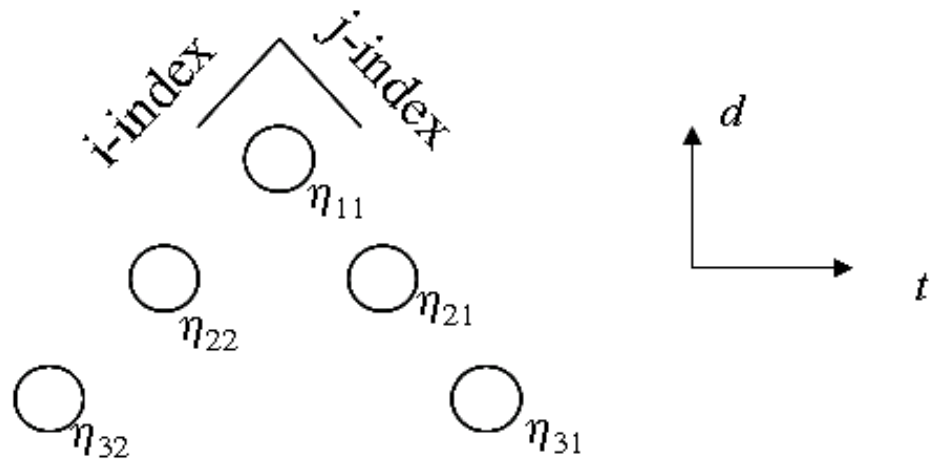

Figure 16. Triangular Formation for Collaborative Hoppers.

$$
\epsilon_{i}^{n+1}=e^{-K} \epsilon_{i}^{n}
$$


And, using a control parameter $K_{r}$ on the relative position of the hoppers, the error expression for the three hoppers are written as

$$
\begin{aligned}
& \epsilon_{1}=\left(\eta_{1}-\eta_{d, 1}\right)+K_{r}\left(\eta_{1}-\eta_{2}-\eta_{d, 12}\right)+K_{r}\left(\eta_{1}-\eta_{3}-\eta_{d, 13}\right), \\
& \epsilon_{2}=\left(\eta_{2}-\eta_{d, 2}\right)+K_{r}\left(\eta_{2}-\eta_{1}-\eta_{d, 21}\right)+K_{r}\left(\eta_{2}-\eta_{3}-\eta_{d, 23}\right),
\end{aligned}
$$

and

$$
\epsilon_{3}=\left(\eta_{3}-\eta_{d, 3}\right)+K_{r}\left(\eta_{3}-\eta_{1}-\eta_{d, 31}\right)+K_{r}\left(\eta_{3}-\eta_{2}-\eta_{d, 32}\right) .
$$

Substituting for $\epsilon^{n+1}$ and $\epsilon^{n}$ from Eq. (36) for all three hoppers gives

$$
\begin{array}{r}
\left(\eta_{1}^{n+1}-\eta_{d, 1}\right)+K_{r}\left(\eta_{1}^{n+1}-\eta_{2}^{n+1}-\eta_{d, 12}\right)+K_{r}\left(\eta_{1}^{n+1}-\eta_{3}^{n+1}-\eta_{d, 13}\right)= \\
e^{-K}\left[\left(\eta_{1}-\eta_{d, 1}\right)+K_{r}\left(\eta_{1}-\eta_{2}-\eta_{d, 12}\right)+K_{r}\left(\eta_{1}-\eta_{3}-\eta_{d, 13}\right)\right] \\
\left(\eta_{2}^{n+1}-\eta_{d, 2}\right)+K_{r}\left(\eta_{2}^{n+1}-\eta_{1}^{n+1}-\eta_{d, 21}\right)+K_{r}\left(\eta_{2}^{n+1}-\eta_{3}^{n+1}-\eta_{d, 23}\right)= \\
e^{-K}\left[\left(\eta_{2}-\eta_{d, 2}\right)+K_{r}\left(\eta_{2}-\eta_{1}-\eta_{d, 21}\right)+K_{r}\left(\eta_{2}-\eta_{3}-\eta_{d, 23}\right)\right]
\end{array}
$$

and

$$
\begin{array}{r}
\left(\eta_{3}^{n+1}-\eta_{d, 3}\right)+K_{r}\left(\eta_{3}^{n+1}-\eta_{1}^{n+1}-\eta_{d, 31}\right)+K_{r}\left(\eta_{3}^{n+1}-\eta_{2}^{n+1}-\eta_{d, 32}\right)= \\
e^{-K}\left[\left(\eta_{3}-\eta_{d, 3}\right)+K_{r}\left(\eta_{3}-\eta_{1}-\eta_{d, 31}\right)+K_{r}\left(\eta_{3}-\eta_{2}-\eta_{d, 32}\right)\right]
\end{array}
$$

Then using Eq. (34) for $\Delta \eta_{1}, \Delta \eta_{2}$, and $\Delta \eta_{3}$, Eq. (40-42) become

$$
\begin{aligned}
& \Delta \eta_{1}\left(1+2 K_{r}\right)-K_{r} \Delta \eta_{2}-K_{r} \Delta \eta_{3}= \\
& \quad \eta_{1}^{n}\left(1+2 K_{r}\right)\left(e^{-K}-1\right)+\eta_{2}^{n} K_{r}\left(1-e^{-K}\right)+\eta_{3}^{n} K_{r}\left(1-e^{-K}\right) \\
& \quad+n_{d, 1}\left(1-e^{-K}\right)+n_{d, 12} K_{r}\left(1-e^{-K}\right)+n_{d, 13} K_{r}\left(1-e^{-K}\right), \\
& \Delta \eta_{2}\left(1+2 K_{r}\right)-K_{r} \Delta \eta_{1}-K_{r} \Delta \eta_{3}= \\
& \quad \eta_{2}^{n}\left(1+2 K_{r}\right)\left(e^{-K}-1\right)+\eta_{1}^{n} K_{r}\left(1-e^{-K}\right)+\eta_{3}^{n} K_{r}\left(1-e^{-K}\right) \\
& \quad+n_{d, 2}\left(1-e^{-K}\right)+n_{d, 21} K_{r}\left(1-e^{-K}\right)+n_{d, 23} K_{r}\left(1-e^{-K}\right),
\end{aligned}
$$

and

$$
\begin{aligned}
\Delta \eta_{3}(1+ & \left.2 K_{r}\right)-K_{r} \Delta \eta_{1}-K_{r} \Delta \eta_{2}= \\
& \eta_{3}^{n}\left(1+2 K_{r}\right)\left(e^{-K}-1\right)+\eta_{1}^{n} K_{r}\left(1-e^{-K}\right)+\eta_{2}^{n} K_{r}\left(1-e^{-K}\right) \\
& +n_{d, 3}\left(1-e^{-K}\right)+n_{d, 31} K_{r}\left(1-e^{-K}\right)+n_{d, 32} K_{r}\left(1-e^{-K}\right) .
\end{aligned}
$$

Hence, solving for the three $\Delta \eta$ 's gives the distance the three hoppers should jump to. As for the case of a single hopper, given a distance to cover, Eq. (22) can be inverted to find the initial velocity components for the required move. The overall motion is governed by $K$ and $K_{r}$.

On Figure 17, the hopper formation achieves the desired configuration and position within a few hops using control parameters $K=1.5$ and $K_{r}=0.5$, for a longitudinal separation distance less than 100 meters. Reducing the control $K_{r}$ makes the triangular path wider before reaching the desired position, which may be desired depending on the application. 
We increase the distance to cover by 3 in the simulation shown in Figure 18. Having a desired position far from the starting point, the dynamics of the asteroid has a more dominant influence on the dynamics and control of the hoppers. In the case shown on Figure 18, the asteroid has stable polar regions. It is clear that the hoppers are "attracted" by the polar regions, making slight curves toward the pole in their trajectory. Even though the following hop attempts to correct the situation, the hoppers 2 and 3 located closer to the polar regions are again attracted to it. This effect could make it impossible for a hopper to reach the desired position and configuration. Note that the paths shown on Figure 17-18 are made of a series of hops from bouncing on the surface, while the hops that are indicated represent controlled jumps.

Hence, the control developed above give good results for small motion, within 100 meters. However, further investigation is needed in order to design an optimal control system valid for longer travel distance and to possibly counteract stronger nonlinear perturbations from the asteroid's dynamics.

As shown in Figure 15, a more rapidly rotating body can have a stable equilibrium at the equator instead of the polar region. In this case, hoppers will tend to stay closer to either one of the principal axes at the equator. However, in this particular case, the analytical model looses its accuracy as even a low energy hop can make a particle, or a hopper, stay in orbit for a few revolutions.

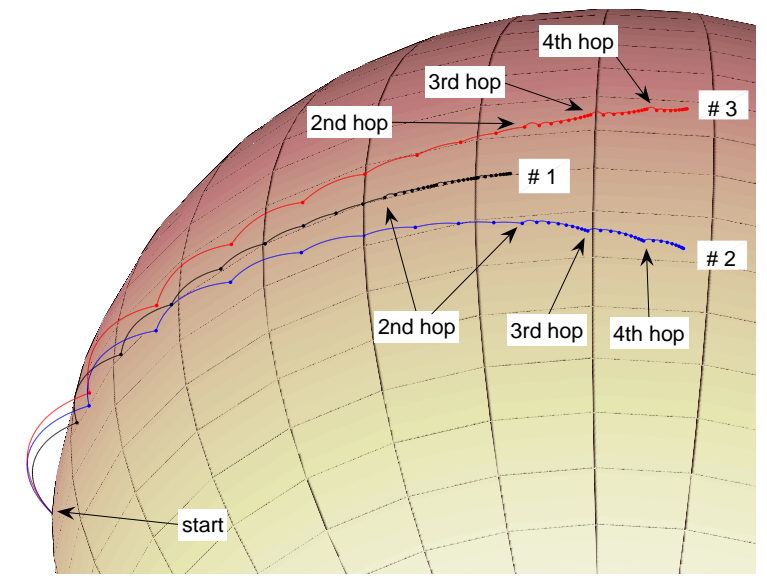

Figure 17. Controlled dynamics of a triangular robot formation with coefficient of restitution and friction factor set to 0.8 and 0.1 , control parameters of $\mathrm{K}=1.5$ and $K_{r}=0.5$, and a longitudinal distance to cover less than 100 meters.

\section{Conclusion}

This paper investigates the dynamics of particles or landers in small body environments, especially for single asteroids and binary asteroid systems. The dynamical models use ellipsoids for modeling single asteroids, and ellipsoid-sphere systems for the binary cases. Surface equilibria have been investigated for rotating ellipsoids, and it is shown that a moving object will stay closer to a stable surface equilibrium while it will move away from an unstable one. In order to counteract the effect of a rotating ellipsoid, a prediction model is developed and used in a control law derived from sliding mode control. It is shown that the control is more accurate for small travel distances as unstable regions may interfere in the direction of motion. 


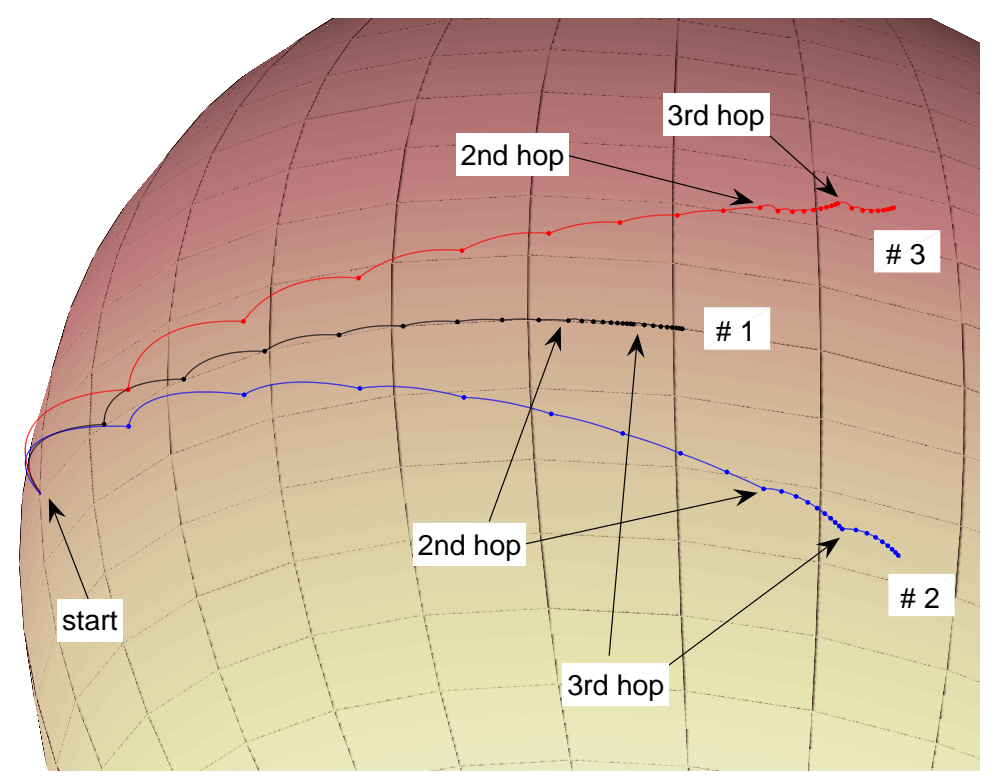

Figure 18. Controlled dynamics of a triangular robot formation with coefficient of restitution and friction factor set to 0.8 and 0.1 , and control parameters $K=1.5$ and $K_{r}=0.5$, with a distance to cover of 200 meters while the polar region is stable.

\section{Acknowledgements}

This research was funded, in part, by a grant from the Jet Propulsion Laboratory/California Institute of Technology Director's Research and Development Fund. Julie Bellerose acknowledges support via a Fellowship from the Natural Sciences and Engineering Research Council of Canada.

\section{References}

${ }^{1}$ J. Bellerose and D.J. Scheeres. Energy and stability in the full two body problem. Celestial Mechanics and Dynamical Astronomy, 100(1):63-91, 2008.

${ }^{2}$ J. Bellerose and D.J. Scheeres. The restricted full three-body problem: Application to binary system 1999 kw4. Journal of Guidance, Control, and Dynamics, 31(1):162-171, 2008.

${ }^{3}$ J.M.A. Danby. Fundamentals of Celestial Mechanics. Willmann-Bell, VA, second edition, 1992.

${ }^{4}$ B.P. Flannery, W.H. Press, S.A. Teukolsky, and W.T. Vetterling. Numerical Recipies in C, The Art of Scientific Computing. Cambridge University Press, second edition, 1996.

${ }^{5}$ V. Guibout and D.J. Scheeres. Stability of surface motion on a rotating ellipsoid. Celestial Mechanics and Dynamical Astronomy, 87:263-290, 2003.

${ }^{6}$ W.M. Kaula. Theory of Satellite Geodesy. Blaisdell Publishing Company, Waltham, Massachusetts, 1966.

${ }^{7}$ A.J. Maciejewski. Reduction, relative equilibria and potential in the two rigid bodies problem. Celestial Mechanics and Dynamical Astronomy, 63:1-28, 1995.

${ }^{8}$ S. Sawai, J. Kawaguchi, D. Scheeres, N. Yoshizawa, and M. Ogasawara. Development of a target marker for landing on asteroids. Journal of Spacecraft and Rockets, 38 (4):601-608, 2001.

${ }^{9}$ D.J. Scheeres. Stability of relative equilibria in the full two-body problem. Annals of the New York Academy of Sciences, 1017:81-94, 2004.

${ }^{10}$ D.J. Scheeres. Relative equilibria for general gravity fields in the sphere-restricted full 2-body problem. Celestial Mechanics and Dynamical Astronomy, 94(3):317-349, 2006.

${ }^{11}$ D.J. Scheeres and S. Augenstein. Spacecraft Motion about Binary Asteroids, volume 116 of Astrodynamics 2003, Part II, Advances in the Astronautical Sciences Series, pages 991-1010. 2003.

${ }^{12}$ D.J. Scheeres, D.D. Durda, and P.E. Geissler. The fate of asteroid ejecta. Asteroid III, pages 162-171, 2002. 
${ }^{13}$ D.J. Scheeres, B.G. Williams, and J.K. Miller. Evaluation of the dynamics environment of an asteroid: Application to 433 eros. Journal of Guidance, Control and Dynamics, 23:466-475, 2000.

${ }^{14}$ T. Yoshimitsu, T. Kubota, I. Nakatani, T. Adachi, and H. Saito. Micro-hopping robot for asteroid exploration. Acta Astronautica, 52:441-446, 2003.

${ }^{15} \mathrm{Z}$. Zheng and A. Girard. Leaderless formation control using dynamic extension and sliding control. IFAC World Congress, 2008.

18 of 18 